\title{
Brain Gene Expression Pattern of Subjects with Completed Suicide and Comorbid Substance Use Disorder
}

\author{
Brenda Cabrera ${ }^{a}$ b Nancy Monroy-Jaramilloc Gabriel Rodrigo Fries ${ }^{d}$ \\ Roberto Cuauhtemoc Mendoza-Morales ${ }^{\mathrm{e}}$ Fernando García-Dolores ${ }^{\mathrm{e}}$ \\ Alejandra Mendoza-Larios ${ }^{\mathrm{e}}$ Carlos Diaz-Otañez ${ }^{\mathrm{e}}$ \\ Consuelo Walss-Bass $^{d}$ David Colin Glahn ${ }^{f, g}$ Patricia Ostrosky-Wegman ${ }^{h}$ \\ Cristobal Fresno ${ }^{i}$ Humberto Nicolini ${ }^{a}$
}

\begin{abstract}
${ }^{a}$ Genomics of Psychiatric and Neurodegenerative Diseases Laboratory, National Institute of Genomic Medicine (INMEGEN), Mexico City, Mexico; ${ }^{b}$ PECEM, Faculty of Medicine, National Autonomous University of Mexico, Mexico City, Mexico; ${ }^{C}$ Department of Genetics, National Institute of Neurology and Neurosurgery, Mexico City, Mexico; ${ }^{\mathrm{d}}$ Department of Psychiatry and Behavioral Sciences, University of Texas Health Science Center at Houston, Houston, TX, USA; ${ }^{e}$ Institute of Forensic Sciences (INCIFO), Mexico City, Mexico; ${ }^{\mathrm{f} D e p a r t m e n t}$ of Psychiatry, Yale School of Medicine, Yale University, New Haven, CT, USA; ${ }^{9}$ Olin Neuropsychiatric Research Center, Institute of Living, Hartford, CT, USA; hiomedical Research Institute, National Autonomous University of Mexico, Mexico City, Mexico; 'Computational Genomics Department, National Institute of Genomic Medicine (INMEGEN), Mexico City, Mexico
\end{abstract}

\section{Keywords}

Postmortem - Dorsolateral prefrontal cortex · Expression study · Microarray · Transcriptomics · Substance use disorder

\begin{abstract}
Background/Aim: Although individuals with substance use disorder (SUD) are at high risk of committing suicide, most studies of postmortem gene expression exclude subjects with SUD due to the potential confounding effect of drugs in the transcriptome. Thus, little is known about the gene expression profile in suicides with SUD. The identification of altered biological processes in suicides with SUD is crucial in the comprehension of the interaction between both pathologies. Methods: We evaluated the gene expression profile in
\end{abstract}

the dorsolateral prefrontal area of suicides and nonsuicides with and without SUD by microarrays. Results: We identified 222 differentially expressed genes, predominately enriched in cell proliferation in the comparison between suicides with and without SUD. When comparing the transcriptome of suicides with SUD to nonsuicides with SUD, we identified 550 differentially expressed genes, mainly enriched in oxidative phosphorylation. Differentially expressed genes $(1,417)$ between suicides and nonsuicides without SUD were detected. Most of them were related to mitochondrial function. Conclusion: Interaction between suicide and SUD seems to influence the expression of genes involved in glial proliferation and glutamatergic neurotransmission. These results highlight, for the first time, that suicides with SUD have a gene expression profile distinct from that of subjects with only one of these disorders.

\section{KARGER}

(C) 2018 S. Karger AG, Basel 


\section{Introduction}

According to the World Health Organization's latest report, more than 800,000 people committed suicide in 2015 , making it the 17th most frequent cause of death worldwide [1]. Suicide rates have been increasing in many countries, including Mexico, where they increased from 3.5 to 5.2 suicides per 100,000 inhabitants between 2000 and 2014 [2]. A first step in decreasing the suicide rate is to identify the factors associated with this behavior. There has been an association between suicidal behavior and certain sociocultural, situational, and individual factors, such as access to health services, interpersonal conflicts, divorce, unemployment, isolation, lack of social support, previous suicide attempt, and presence of mental disorders $[1,3]$.

Several postmortem studies using a psychological autopsy have confirmed the association between suicide and the presence of mental disorders. The well-known review of Fleischmann et al. [4] reported that $88.6 \%$ of the suicide victims suffered from a mental disorder, where mood disorders were the most frequent diagnosis (42.1\%), followed very closely by substance use disorder (SUD) [4]. Similar rates of mental illnesses among individuals who successfully committed suicide have been recently reported $[5,6]$.

It is estimated that, compared to the general population, subjects with alcohol abuse are almost ten times more likely to commit suicide, whereas people with abuse of other substances are approximately fourteen times more likely to commit this act [7]. Dependence on several substances such as alcohol, inhalants, heroin [8], and tobacco [9] have also been associated with suicidal behavior. Although subjects with SUD are at an increased risk for suicidal behavior, most postmortem suicide gene expression studies have excluded this group of patients [10]. Therefore, little is known about the molecular basis of the association between SUD and suicidal behavior. A recent study by Gandal et al. [11] (2018) evaluated the transcriptome of five mental disorders; autism, schizophrenia, bipolar disorder, major depression, and alcohol use disorder (AUD), where they observed a lack of overlap between the AUD and the other mental disorders. This result suggests that a different gene expression profile exists in suicides with SUD compared to subjects with other mental pathologies.

The prefrontal cortex is the brain region responsible for decision making, inhibition and short-term memory, functions known to be altered in subjects with suicidal behavior [12, 13]. Structural and functional changes in the prefrontal cortex, specifically the dorsolateral prefrontal cortex, as well as alterations in cognitive abilities associated with the prefrontal region, have been identified in subjects with suicidal behavior and in subjects with SUD [14]. Therefore, it is of particular interest to evaluate gene expression in this brain region in suicidal subjects with and without SUD, in order to have a better understanding of the interaction between suicide and SUD. The identified genes could be used as potential biomarkers of suicidal behavior in subjects with SUD and ultimately for the implementation of novel treatments for this population. In this preliminary study, we compared the gene expression profile in the dorsolateral prefrontal area of subjects who committed suicide with and without SUD to the transcriptome of subjects with and without SUD who died due to a cause other than suicide.

\section{Materials and Methods}

\section{Subjects and Brain Samples}

Postmortem brain samples were obtained from the Institute of Forensic Sciences (INCIFO) in Mexico City, Mexico, from subjects who committed suicide and subjects with sudden death in 2016 in Mexico City. Suicide completers are defined as those individuals whose causes of death were self-inflicted injuries, which correspond to X60-X84 codes of the International Classification of Diseases, 10th Revision (ICD-10). The nonsuicidal subjects presented sudden, not self-inflicted death (for example, a car accident) without a period of prolonged agonal state or prolonged illness. The Ethics Committee for Human Research at the National Institute of Genomic Medicine approved this research.

Fresh brain tissue samples from the dorsolateral prefrontal cortex (Brodmann area 9) were dissected using the middle frontal gyrus and the precentral sulcus anatomical references and stored in RNAlater, an RNA stabilization reagent (Qiagen, Singapore) at $-80^{\circ} \mathrm{C}$ until its use. The postmortem interval (PMI) represents the time interval between the estimated time of death and the sample collection, which ranged from 06:50 to 29:30 h. A toxicology test for detecting substance consumption at the time of death was performed in both peripheral blood and brain tissue.

Subjects were psychiatrically characterized by a diagnostic estimation based on data obtained through from the legal medical records, which contain (i) declarations from the police, relatives, and witnesses, (ii) demographic information, (iii) acute and chronic stressful life situations, (iv) autopsy and toxicological reports, and (v) clinical records in case the individual received medical attention. A consensus diagnosis, based on the Diagnostic and Statistical Manual of Mental Disorders, Fifth Edition (DSM-5) criteria, was achieved between a pathologist, a psychologist, and a psychiatrist, and then reviewed and confirmed by an independent experienced psychiatrist. The complete methods for diagnostic estimation are described elsewhere [15].

Sixty-six samples were collected, considering the following experimental groups: 23 suicide completers with comorbid SUD (Suicidal SUD+ group), 20 suicide completers without SUD (Sui- 
Table 1. Demographic, clinical, and suicide subjects' characteristics

\begin{tabular}{|c|c|c|c|c|c|}
\hline \multirow[b]{2}{*}{ SUD } & \multicolumn{2}{|l|}{ Suicides } & \multicolumn{2}{|l|}{ Nonsuicides } & \multirow[t]{2}{*}{$p$ value } \\
\hline & + & - & + & - & \\
\hline Subjects & 23 & 20 & 9 & 14 & \\
\hline Age, years & $31.95 \pm 17.43$ & $32.8 \pm 15.19$ & $30.88 \pm 7.04$ & $31.78 \pm 19.51$ & 0.645 \\
\hline Gender (M:F) & $21: 2$ & $12: 8$ & $8: 1$ & $8: 6$ & 0.028 \\
\hline PMI, h & $14.91 \pm 3.91$ & $15.03 \pm 4.81$ & $17.76 \pm 7.07$ & $16.84 \pm 4.44$ & 0.128 \\
\hline AUD & 17 & 0 & 8 & 0 & \\
\hline Positive result in toxicology test & 16 & 3 & 4 & 0 & \\
\hline Cause of death ${ }^{\mathrm{a}}$ & $19 / 0 / 2 / 1 / 1 / 0 / 0$ & $16 / 1 / 1 / 1 / 1 / 0 / 0$ & $1 / 2 / 0 / 3 / 2 / 1 / 0$ & $4 / 4 / 0 / 2 / 0 / 1 / 4$ & \\
\hline
\end{tabular}

Values indicate number of subjects unless otherwise indicated. Continuous data is presented as mean \pm standard deviation. + , positive; -, negative; SUD, substance use disorders; M, male; F, female; PMI, postmortem interval; AUD, alcohol use disorder. ${ }^{a}$ Asphyxia/ gunshot/intoxication/trauma/puncture wound/traffic accident/shock. Two-tailed $p$ value test is described in Methods.

cidal SUD- group), 9 subjects with SUD whose cause of death was not suicide (Nonsuicidal SUD+ group), and 14 nonsuicide subjects without SUD (Nonsuicidal SUD- group); the latter two groups were named as nonsuicides for the purposes of this study. Demographic, clinical, and suicide subjects' characteristics are described in Table 1. Categorical variables between experimental groups, such as gender, were assessed by Fisher's exact test, and continuous variables, for instance age and PMI, using analysis of the variance (ANOVA) tests. The interactive comparison between the experimental groups is illustrated in Figure 1.

\section{RNA Isolation}

RNA isolation was performed using the RNeasy kit from Qiagen $^{\mathrm{TM}}$ following the manufacturer's instructions. The purity of the extracted RNA was evaluated on the NanoDrop1000 spectrophotometer (Thermo Scientific, Waltham, MA, USA) and its integrity by electrophoresis with $1 \%$ agarose gel. Subsequently, the RNA integrity number of the samples was evaluated using the Agilent Bioanalyzer. Only samples with an RNA integrity number $>7$ were further processed.

\section{Microarray Detection}

The gene expression of the 66 samples was analyzed using the Illumina HumanHT-12 v4 BeadChip microarray, which contains 47,231 probes. RNA samples were labeled into biotinylated cRNAs with the TargetAmp ${ }^{\mathrm{TM}}$-Nano Labeling Kit (Epicentre), followed by hybridization into the BeadChips according to the manufacturer's protocol. The BeadChips were scanned on an iScan Microarray Scanner (Illumina) immediately after the protocol.

\section{Microarray Analysis}

For data pre-processing the 47,231 probes contained in the microarray were annotated so that each one was assigned their respective EntrezID and gene symbol with the Bioconductor "IlluminaHumanv4.db" package [16]. Then, raw probe intensities were background corrected with the "normexp" method with "mle" maximum likelihood approximation in the "limma" package [1719]. Subsequently, control, ERCC, and unannotated probes (without a valid EntrezID number) were filtered out. Low detection probes, namely those with a detection $p$ value $>0.05$ and those not

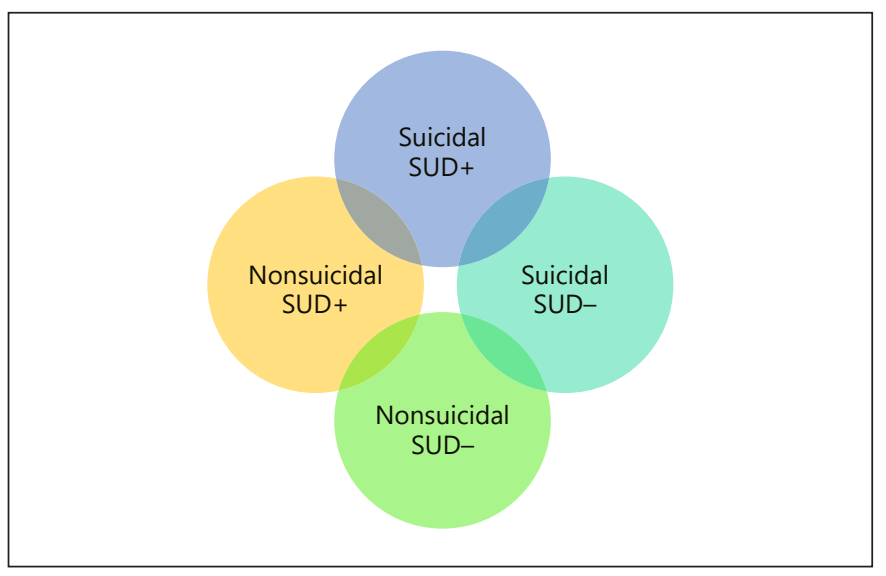

Fig. 1. Venn diagrams for the interactive comparison between experimental groups. In this study, the gene expression profile of Brodmann area 9 of suicides with substance use disorder (Suicidal SUD+) and nonsuicides without SUD (Nonsuicidal SUD) were compared to suicides without SUD (Suicidal SUD-) and nonsuicides with SUD (Nonsuicidal SUD+). The complete lists of differentially expressed genes in these comparisons are shown in the online supplementary material.

present in at least $50 \%$ of arrays in each experimental group, were removed.

Gene expression values were normalized to compensate for systematic technical differences between the arrays by the quantile method and $\log _{2}$-transformed [20]. Replicate probes within the arrays were summarized and replaced with their average value. To visualize the data and evaluate possible batch effects, we performed a principal component analysis where we could detect a batch effect associated with an unknown source of variation. A multivariate analysis with the ARSyN package was executed to remove such an effect [21].

The resulting data comprises a $2 \times 2$ factorial experimental design. The first factor is whether the individual committed suicide 


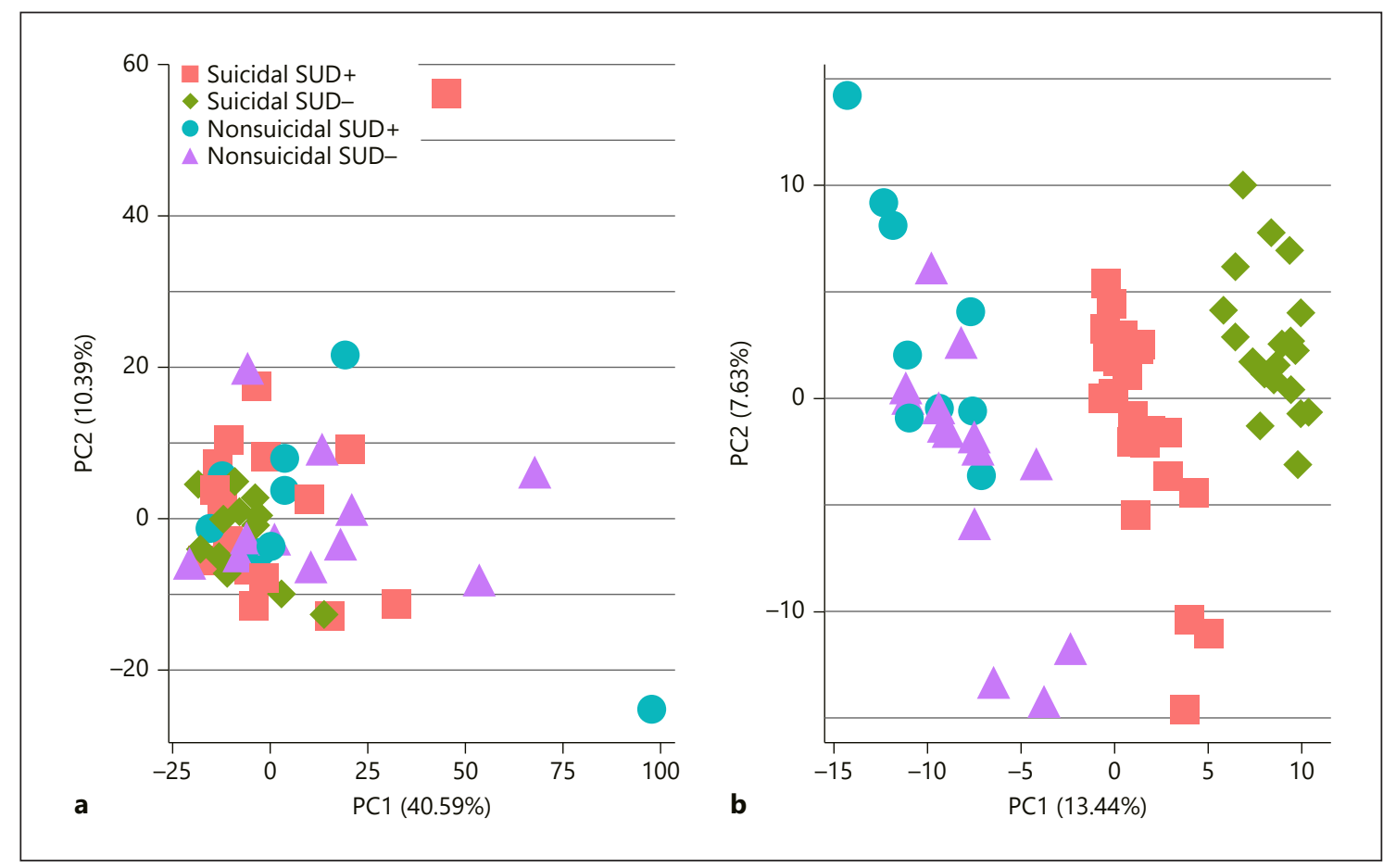

Fig. 2. Principal component analysis (PCA) scatter plot of the studied subjects. a Using quantile-normalized gene expression levels. b After multi-dimensional normalization using ARSyN. It is worth noting that in a the experimental groups are confounded due to an excess of uncontrolled variability (batch effect). This effect was corrected in $\mathbf{b}$, where the first two principal components (PC1 and PC2) demonstrated a clear separation of these groups. Experimental groups: suicide (Suicidal), nonsuicide (Nonsuicidal), with or without substance use disorder (SUD+/SUD-).

or not. The second factor is the absence or presence of SUD. The data was analyzed using a gene by gene independent linear model using the Bioconductor "limma" package $[17,18]$ according to equation (1):

$$
y_{i j k}=\mu+\alpha I_{\text {Suicide }_{i}}+\beta \gamma I_{\text {Substance }_{j}}+\gamma I_{\text {Suicide }_{i}} \times I_{\text {Substance }_{j}}+\epsilon_{i j k}
$$

where, $y_{i j k}$ is the $\log _{2}$ gene expression level for the $\mathrm{i}$-th suicide level, under the $\mathrm{j}$-th SUD level, in the $\mathrm{k}$-th biological replicate; $\mu$ is the global average gene expression; $\alpha, \beta$, and $\gamma$ are the suicide and SUD coefficients, respectively; $I_{\text {Suicide }_{i}}$ and $I_{\text {Substance }_{j}}$ are the indicator dummy functions ( 0 or 1 ), for $i$-th suicide and $j$-th SUD levels, respectively; $\varepsilon_{\mathrm{ijk}} \sim \mathrm{N}\left(0, \sigma^{2}\right)$ is the random error which is normally distributed with zero expected value and variance equal to $\sigma^{2}$ for the $\mathrm{i}$-th suicide, $\mathrm{j}$-th substance, and $\mathrm{k}$-th biological replicate respective levels.

Principal effect and corresponding double interaction assessments were carried out using Type-III sum of squares. Biological marginal hypothesis tests between specific experimental groups were performed using a two-tailed $\mathrm{F}$ test using the complete estimated linear model parameters. $p$ values associated with the differentially expressed genes were corrected for multiple comparisons using the Benjamini-Hochberg false discovery rate method [22]. For the purposes of this study, the significant level was set to 0.001 . Visualization of the results from each comparison was car-

Gene Expression in Suicide and Substance Use Disorder ried out by means of heatmaps using "gplot" [23]. The data preprocessing and processing were executed in $\mathrm{R}[24]$.

\section{Functional Annotation Analysis}

The Database for Annotation, Visualization and Integrated Discovery (DAVID), version 6.8, was used for over-representation analysis $[25,26]$. The annotation categories were restricted to use: Kyoto Encyclopedia of Gene and Genomes (KEGG), Gene Ontology (GO), and Reactome. The up- and downregulated genes were analyzed separately in DAVID, since this approach is more powerful for finding significant functional pathways documented in the databases [27]. Only the resulting 6,862 transcripts were used as background reference. Biological pathways with a modified Fisher exact $p$ value $<0.05$ (EASE score) were considered enriched [28]. In addition, Ingenuity ${ }^{\circledR}$ Pathway Analysis software (IPA ${ }^{\circledR}$; QIAGEN Redwood City) was used to complement the analysis [29].

\section{Results}

\section{Subjects and Brain Samples}

According to the demographic, clinical, and suicide subjects' characteristics presented in Table 1, no signifi- 


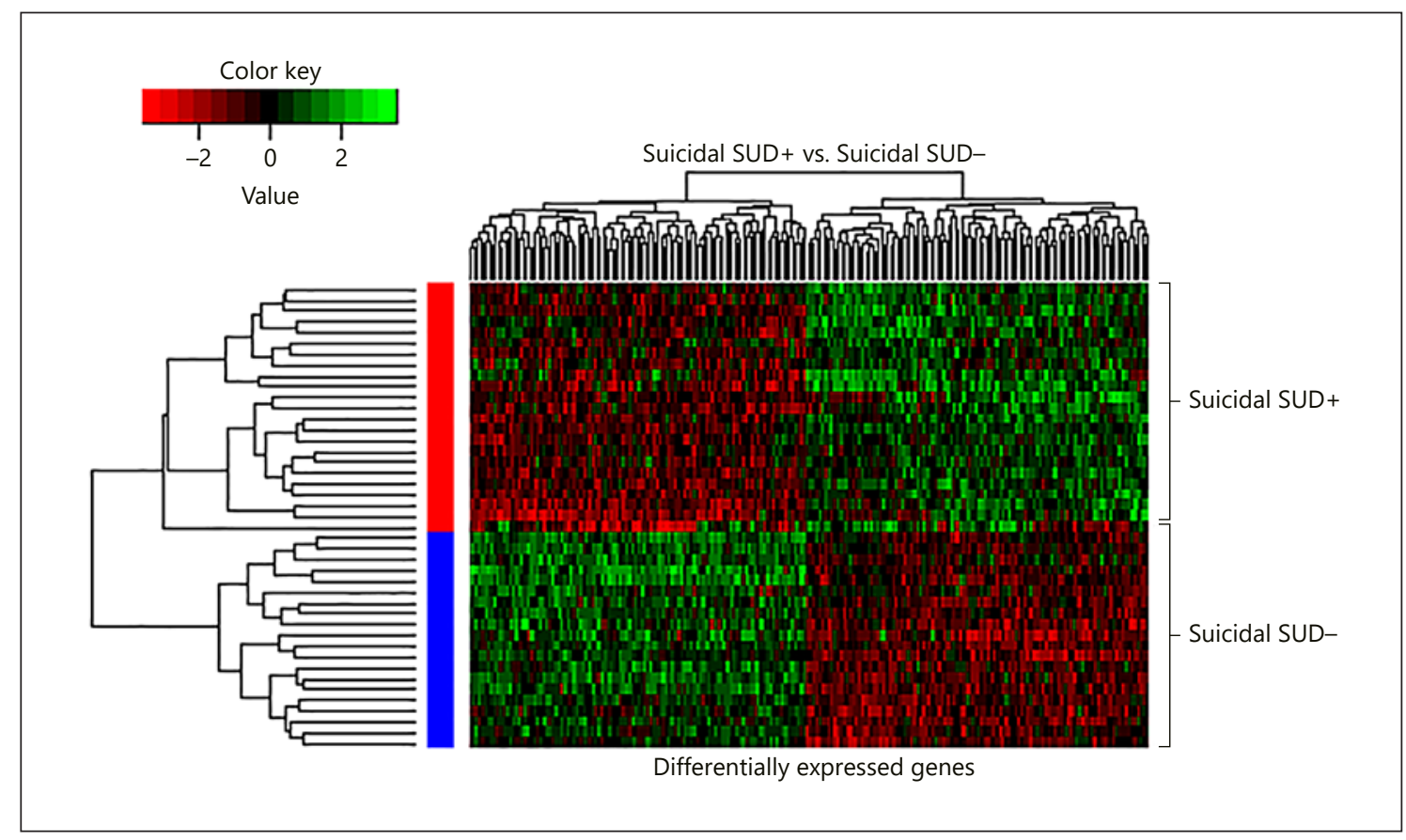

Fig. 3. Substance use comparison heatmap for suicide subjects. The 222 differentially expressed genes (in columns) between suicide subjects with substance use disorder (Suicidal SUD+) and suicide subjects without SUD (Suicidal SUD-), with red and blue color, respectively (in rows). Gene expression levels are depicted in red-green spectrum for down-/upregulated genes.

cant differences were observed between the experimental groups in terms of age ( $p$ value $=0.645)$ and PMI ( $p$ val$\mathrm{ue}=0.128)$. However, there was an association between gender and experimental group ( $p$ value $=0.028$ ). In this context, more men than women successfully committed suicide across all the groups. This fact is consistent with previous reports in the Mexican population, where it is known that the majority of those who commit suicide or suffer from SUD belong to the male gender [2].

In the suicide with SUD group, $73.91 \%(20 / 23)$ of the subjects had AUD, whereas in the nonsuicidal group with SUD, $88.88 \%(8 / 9)$ of the subjects had AUD. From the subjects who obtained a positive result in the toxicology test from the suicide with SUD group, $87.5 \%$ were positive for alcohol only and $12.5 \%$ were positive for alcohol and cocaine. Subjects from the other groups were only positive for alcohol.

The quantile-normalized gene expression levels were inspected for batch effects using principal component analysis (PCA) over the subjects (Fig. 2a). Together, the first two components (PC1 and PC2) represented 50.98\% of the total variability. Unfortunately, the experimental groups were mixed together whereas one would have ex- pected that they clustered. However, after applying ARSyN (Fig. 2b), a noteworthy separation along the PC1 axis formed three clusters: (i) Suicidal SUD- subjects, (ii) Suicidal SUD+ subjects, and (iii) nonsuicides (Nonsuicidal SUD+ and Nonsuicidal SUD-), using only $13.44 \%$ of the total gene expression variance.

\section{Suicidal SUD+ versus Suicidal SUD-}

In the comparison between subjects who committed suicide and had SUD $(n=23)$ and subjects who committed suicide without SUD $(n=20), 222$ differentially expressed genes were detected, yielding 112/110 genes up-/ downregulated, respectively. Visual inspection over the corresponding heatmap showed that the two experimental groups clustered together (Fig. 3). The complete list of differentially expressed genes in this comparison is shown in the online supplementary material (for all online suppl. material, see www.karger.com/doi/10.1159/000493940).

The functional analysis results of the downregulated genes showed that GO terms were significantly enriched in cation transport, neuron part, signaling, synapse, immune response receptors, behavior, learning or memory, and cellular respiration. The upregulated genes showed 


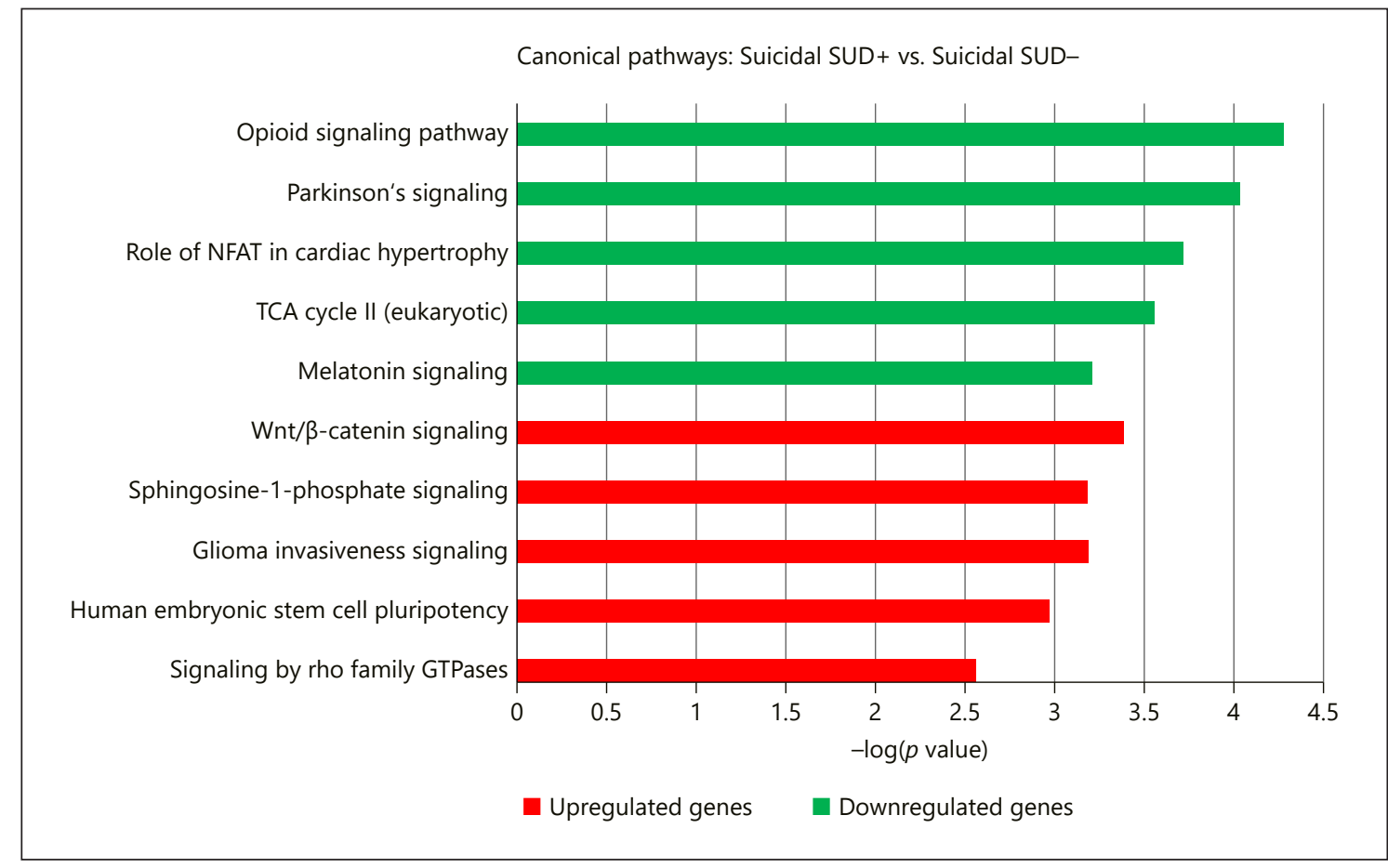

Fig. 4. Top 5 enriched canonical pathways from IPA. Canonical pathways related to upregulated genes (red bars) and downregulated genes (green bars) detected when comparing suicides with substance use disorder (Suicidal SUD+) to suicides without SUD (Suicidal SUD-).

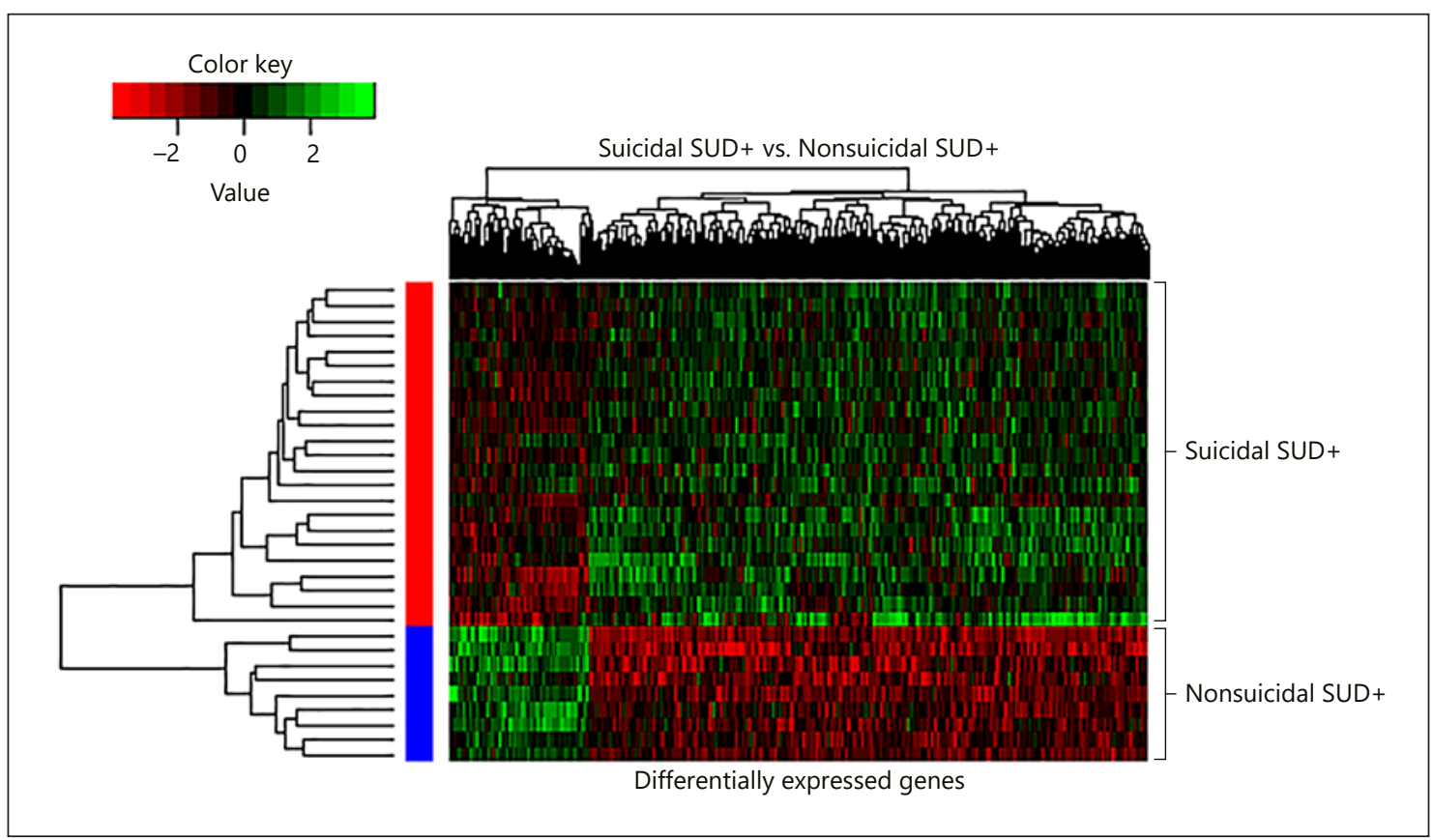

Fig. 5. Suicide comparison heatmap for subjects with substance use disorder (SUD). The 550 differentially expressed genes (in columns) between suicides with SUD (Suicidal SUD+) and nonsuicidal subjects with SUD (Nonsuicidal SUD+), with red and blue color, respectively (in rows). Gene expression levels are depicted in redgreen spectrum for down-/upregulated genes. 


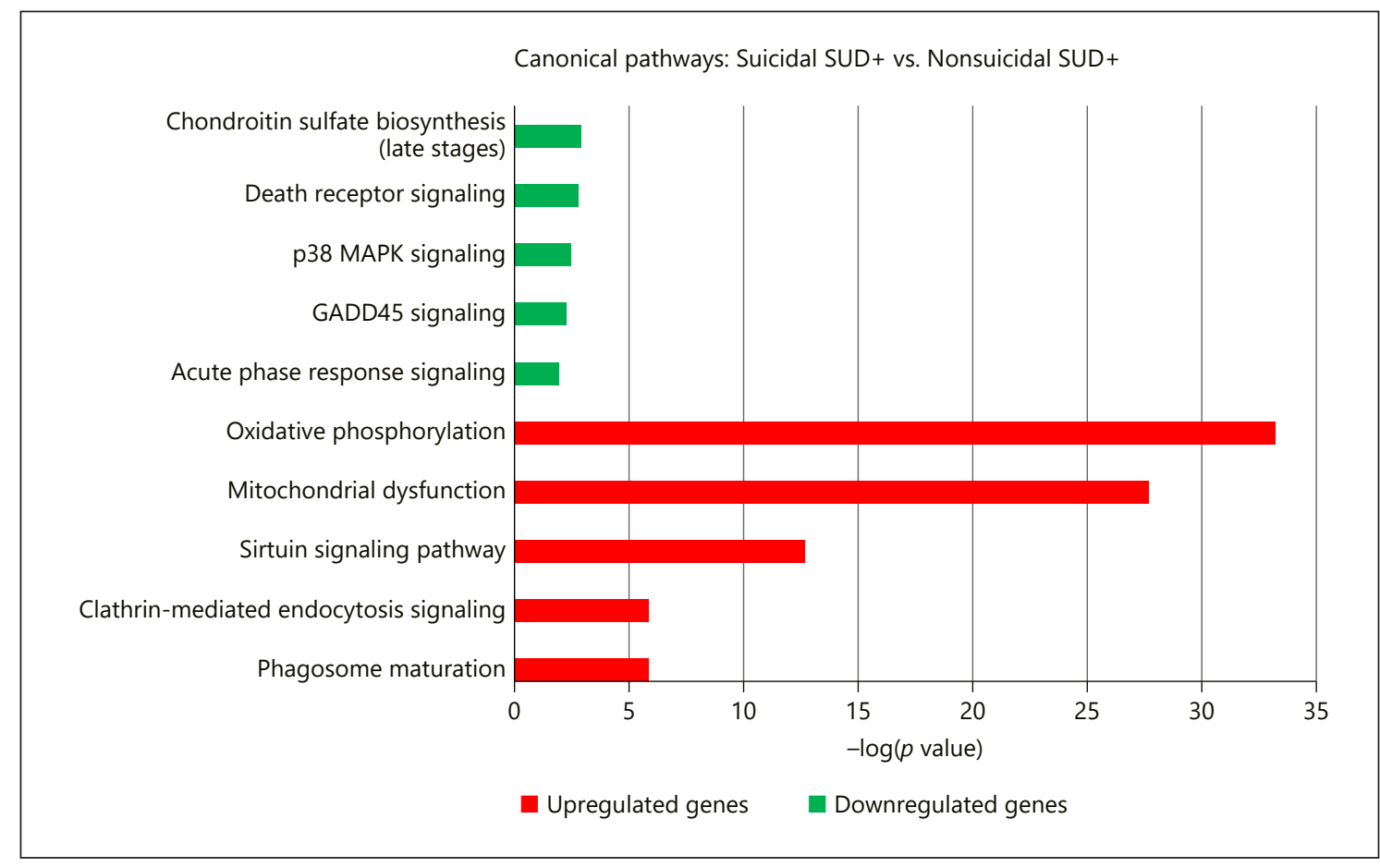

Fig. 6. Top 5 enriched canonical pathways from IPA. Canonical pathways related to upregulated genes (red bars) and downregulated genes (green bars) detected when comparing suicides with substance use disorder (Suicidal SUD+) to nonsuicides with SUD (Nonsuicidal SUD+).

enrichment for GO terms related to epithelium development, cell adhesion, regulation of nervous system development, regulation of gene expression, and phosphatidylinositol-mediated signaling. The complete list of related GO terms is available in online supplementary Table 1.

The joint functional analysis, including both up- and downregulated genes, indicated that the genes identified as differentially expressed genes between suicidal with SUD (Suicidal SUD+) and suicidal without SUD (Suicidal SUD-) groups were associated with cell differentiation, cell death, cell adhesion, and neurogenesis. Since cell differentiation was the GO term associated with the highest percentage of genes, we further investigated terms related to this GO category in this comparison and found "neuron and glial cell differentiation" with a significant $p$ value. The complete list of related GO terms resulting from this joint analysis is available in online supplementary Table 2.

Moreover, the top five canonical pathways identified by IPA ranked by their $p$ value related to the up- and downregulated genes are illustrated in Figure 4. The top ten canonical pathways derived from the analysis of all differentially expressed genes is available in online supplementary Figure S1.

\section{Suicidal SUD+ versus Nonsuicidal SUD+}

The gene expression profile for suicides with SUD $(n=23)$ with respect to suicides without SUD $(n=9)$ showed 550 differentially expressed genes, of which 438 were upregulated and 112 downregulated. Visual inspection over the corresponding heatmap showed that the two experimental groups clustered together (Fig. 5). The complete list of differentially expressed genes in this comparison is in the online supplementary material.

In this comparison, the downregulated genes showed enriched GO terms related to signal peptides, receptor, zinc finger proteins, and metabolic processes. The upregulated genes were associated with the mitochondrial membrane, antigen processing and presentation, and cytoskeleton. The complete list of GO terms is available in online supplementary Table 3.

When analyzing the complete list of differentially expressed genes between suicidal individuals with SUD and nonsuicidal individuals with SUD, we found that they were associated with immune response, oxidative phosphoryla-
Cabrera et al. 


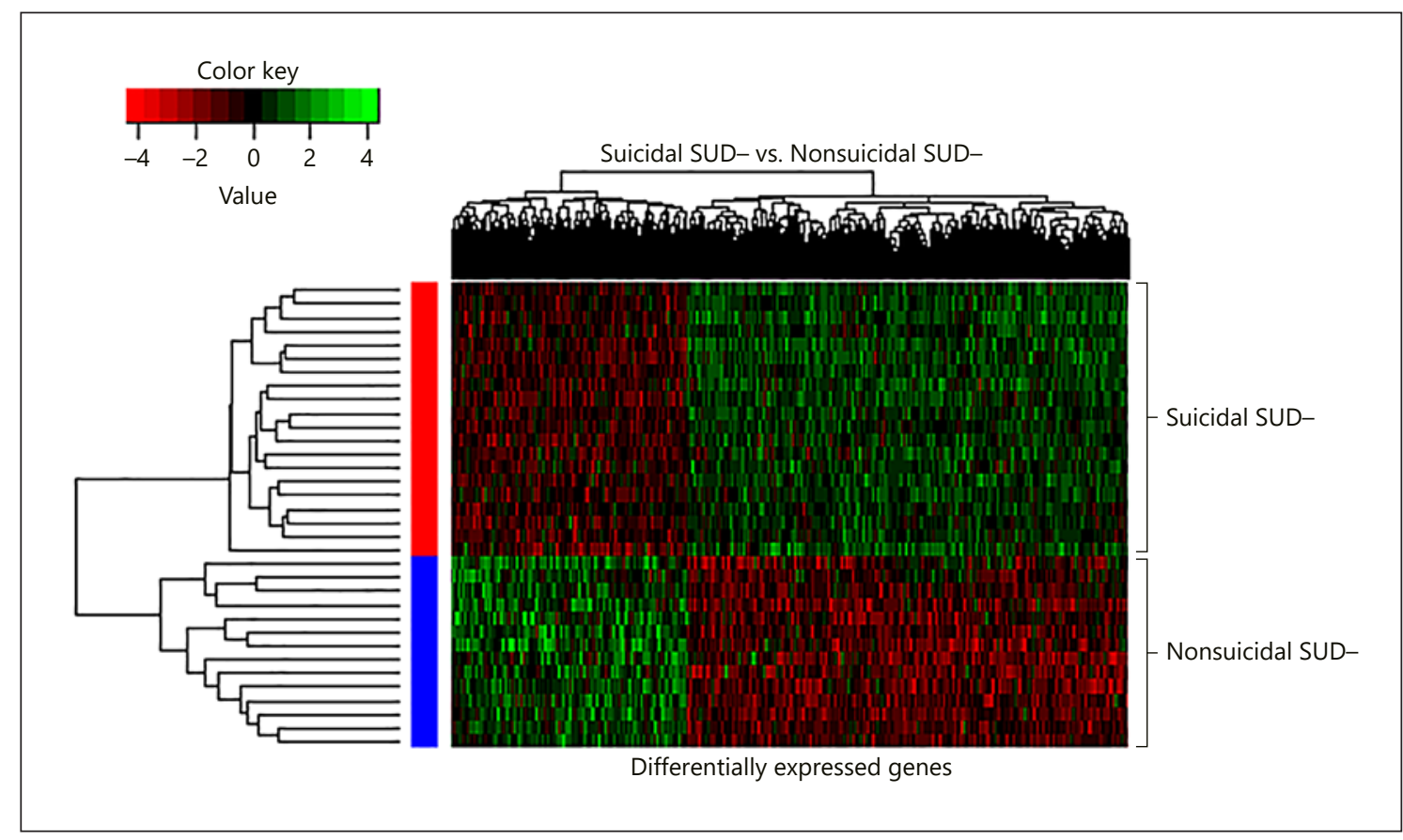

Fig. 7. Suicide comparison heatmap for subjects without substance use disorder (SUD). The 1,417 differentially expressed genes (in columns) between suicides without SUD (Suicidal SUD-) and nonsuicidal subjects without SUD (Nonsuicidal SUD-), with red and blue color, respectively (in rows). Gene expression levels are depicted in red-green spectrum for down-/upregulated genes.

tion, Parkinson's disease, and mitotic cell cycle phase transition. The complete list of GO terms obtained from this joint analysis is available in online supplementary Table 4 .

The top five canonical pathways identified by IPA ranked by $p$ value related to differentially expressed genes are illustrated in Figure 6. The top ten canonical pathways resulting from the joint analysis of differentially expressed genes identified in this comparison are available in online supplementary Figure S2.

\section{Suicidal SUD- versus Nonsuicidal SUD-}

In the comparison between suicides without SUD $(n=$ $20)$ and nonsuicides without SUD $(n=14), 1,417$ differentially expressed genes were detected, yielding 923/494 genes up-/downregulated, respectively. The corresponding heatmap showed that the two experimental groups clustered together (Fig. 7). The complete list of differentially expressed genes identified in this comparison is available in the online supplementary material.

In this comparison, the downregulated genes exhibited enriched GO terms associated with cell communication, regulation of gene expression, neurogenesis, and programmed cell death. Regarding the last term, it is noteworthy that it includes genes of both positive and negative regulation of this process. The upregulated genes were related to mitochondria, programmed cell death, ion transport, and neuron projection. The complete list of GO terms from this analysis is available in online supplementary Table 5 .

In the joint functional analysis, with both up- and downregulated genes, we observed that most of the genes identified as differentially expressed genes between suicidal without SUD and nonsuicidal without SUD groups were enriched in GO terms related to mitochondrion, regulation of cell communication, programmed cell death, and neuron differentiation (online suppl. Table 6).

The top five canonical pathways identified by IPA ranked by $p$ value related to differentially expressed genes are illustrated in Figure 8. The top ten canonical pathways resulting from the analysis of all differentially expressed genes identified in this comparison is available in online supplementary Figure S3.

\section{Nonsuicidal SUD+ versus Nonsuicidal SUD-}

When comparing nonsuicides with SUD to nonsuicides without SUD, we identified only one gene to be 


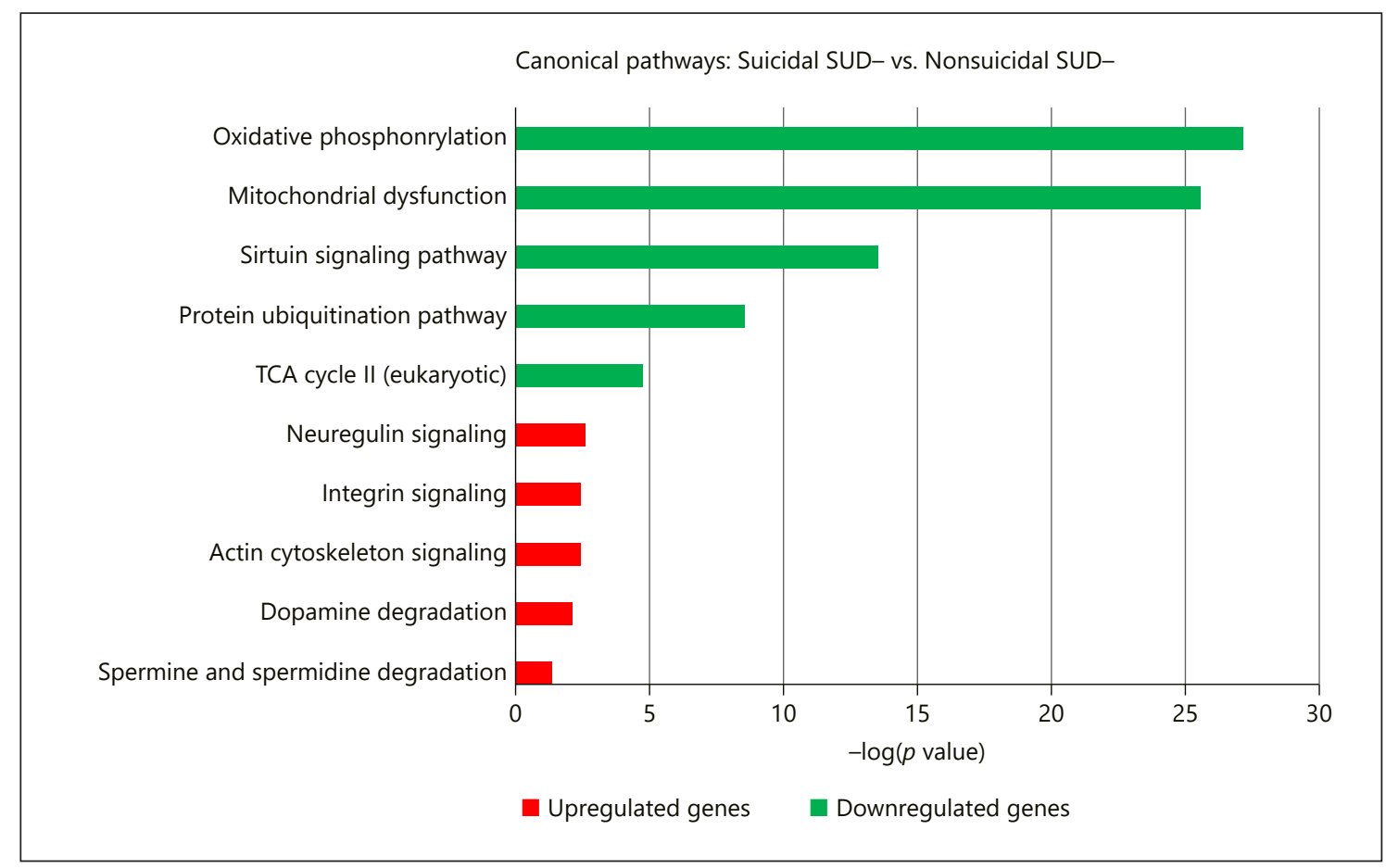

Fig. 8. Top 5 enriched canonical pathways from IPA. Canonical pathways related to upregulated genes (red bars) and downregulated genes (green bars) detected when comparing suicides with substance use disorder (Suicidal SUD-) to nonsuicides with SUD (Nonsuicidal SUD-).

downregulated, LOC285074, which encodes for the anaphase promoting complex subunit 1 pseudogene.

\section{Interaction between Suicide and SUD}

The interaction term (suicide by SUD) exhibited influence in the expression of 55 genes; 26 of these genes were downregulated and 29 were upregulated. The functional enriched GO terms related to these genes are shown in Table 2. Results of IPA for such genes are available in online supplementary Figure S4.

\section{Discussion}

To the best of our knowledge, this is the first transcriptome-level study comparing gene expression profiles in the dorsolateral prefrontal cortex of suicides with SUD and suicides without this disorder, as SUD has been considered in most studies as a confounder and hence excluded from the analysis. Two studies identified multiple probesets whose expression was influenced by alcohol or cocaine use in the ventral prefrontal cortex [30], limbic system, and posterior cingulate gyrus [31] in suicides with major depression of French-Canadian origin. One of these studies [32] included drug or alcohol use in their statistical models, without finding an association between substance abuse and the expression of differentially expressed genes in Brodmann area 46. The other examined the effects of alcohol on genes identified as differentially expressed in a larger French-Canadian sample and in a murine model of acute and chronic alcohol use, discarding the fact that the pattern of gene expression observed in the study was due to the use of substances [33]. Most of the studies have either excluded subjects based on toxicology reports, which only provide information about the use of certain drugs at the moment of death, or ignored the issue altogether [10].

\section{Suicidal SUD+ versus Suicidal SUD-}

When comparing suicides with SUD to suicides without SUD, an evident overexpression of genes participating in pathways involved in the regulation of cellular proliferation and migration, such as Rho family GTPases, glioma invasiveness signaling, and SP1 signaling, known for its determinant role in cell survival and proliferation, was identified $[34,35]$. Parkinson's signaling is one of the
Cabrera et al. 
Table 2. GO functional terms associated with the genes participating in the interaction between SUD and suicide

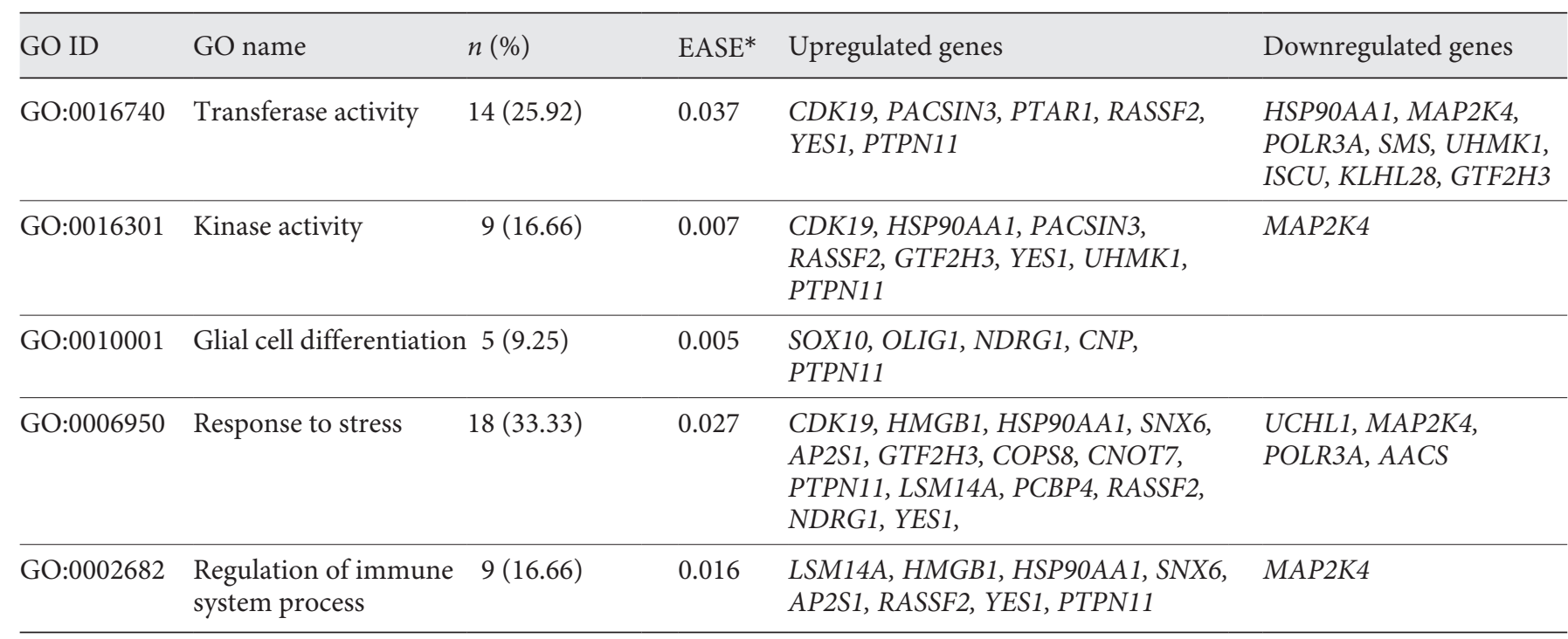

GO, Gene Ontology; SUD, substance use disorder. * Modified Fisher exact $p$ value.

enriched pathways for the downregulated genes in this comparison, which include the pro-apoptotic genes CYCC, UCHL1, and SNCA [36]. Of note, the abnormal expression of genes involved in cell proliferation and its regulation, such as $M A P K$, suggests that suicides with SUD may have an increased cell proliferation, which could lead to structural abnormalities in the prefrontal cortex of suicides with SUD and lead to an impaired function of this brain area.

This probable increased cell proliferation, if corroborated by other studies, could lead to a higher cell density which would be different from the decreased neuronal and glial density in the prefrontal cortex reported for subjects with AUD in suicides [37-39]. Lower neuronal density has been reported in Brodmann area 9 [40]; however, the glial density has only been evaluated in the white matter of this brain area, where abnormal density of microglia was found in subjects who committed suicide [41].

Among the genes identified as differentially expressed between suicides with SUD and suicides without SUD are SLC1A2, SLC1A3, and GLUD1, which encode for proteins involved in glutamate metabolism. The first two genes are transporters of this neurotransmitter; GLUD 1, glutamate dehydrogenase, catalyzes the oxidative deamination of glutamate. The alteration of glutamatergic neurotransmission in subjects with suicidal behavior is well documented; however, an interesting finding from our

Gene Expression in Suicide and Substance

Use Disorder study is the overexpression of these genes in suicide subjects with SUD, contrary to previous postmortem studies of suicide with depression [42] and bipolar disorder [43] in the same brain area. This overexpression could be due to the chronic exposure to ethanol in most of the suicide subjects with SUD in our sample, as alterations in glutamatergic neurotransmission have been reported in subjects with alcohol dependence [44] and in animal models of alcoholism [45]. Our finding suggests that suicide with SUD present alterations in glutamatergic neurotransmission, and these abnormalities are different from those found in suicide without this comorbidity.

Another interesting finding is the overexpression of genes that encode for connexins 30 and 43 in suicide with SUD. Reduced expression of these genes has been reported in suicide cases [33] in addition to a reduced expression of its protein in subjects with alcohol dependence [46]. The finding that the expression of genes involved in glutamatergic neurotransmission and genes for connexins 30 and 43 are in the opposite direction to that previously reported in suicide with other psychiatric pathologies indicates that the presence of SUD leads to unique changes in gene expression in suicide subjects. If replicated in other populations, and corroborated by functional analysis, these findings could have important therapeutic implications, leading to treatment strategies focused on specific alterations in patients with SUD who could be at risk of committing suicide. 
Suicidal SUD+ versus Nonsuicidal SUD+

An overexpression of genes involved in oxidative phosphorylation and mitochondrial function was detected in suicides with SUD compared to nonsuicide subjects with SUD, which may indicate an increased energy demand with a potential impact on synaptic and neuronal function. Since mitochondrially formed oxidants activate several signaling pathways related to apoptosis [47], this GO term was associated with the differentially expressed genes identified in this comparison, as expected.

In addition, mitochondrial function is crucial in neural migration, an especially relevant process, given the nature of the tissue studied [48-50]. Other canonical pathways associated with the differentially expressed genes from this comparison are the clathrin-mediated endocytosis [51] and chondroitin sulfate synthesis [52], which are also implicated in neural migration. This suggests a dysregulation in this function in suicides with SUD, perhaps as part of a synaptic refinement process [53].

\section{Suicidal SUD- versus Nonsuicidal SUD-}

As in the previous comparison, we detected an overexpression of genes involved in mitochondrial function. This observation is interesting, since both comparisons are between suicidal and nonsuicidal subjects in the presence of SUD and in the absence of this disorder, respectively. This suggests that the increased expression of mitochondrial genes could be relevant in the pathophysiology of suicide independently of the comorbidity with SUD.

Pantazatos et al. [54] reported an increased expression of genes involved in "DNA-dependent ATPase activity" in suicides compared to nonsuicides ( $p<0.1$ corrected). Therefore, we anticipate that future studies focusing on the mitochondrial function of subjects with suicidal behavior may help to clarify the role of this process in suicide.

\section{Nonsuicidal SUD+ versus Nonsuicidal SUD-}

When we compared the gene expression profile of nonsuicides with SUD to nonsuicides without SUD we detected the downregulation of LOC285074, which encodes for a pseudogene. Our criteria for considering a transcript as differentially expressed between groups was probably especially strict in order to detect more differences in this comparison. In the future, with the knowledge generated from other studies, we may be able to confirm if this pseudogene is relevant in the SUD pathology in nonsuicidal subjects.

Of note, results obtained from studies in neurodegenerative diseases suggest that pseudogenes and other non- coding transcripts could participate in the regulation of gene expression through competition for miRNA-occupied sites [55].

\section{Interaction between Suicide and SUD}

In addition to finding an overexpression of genes involved in cell proliferation in the comparison between suicides with SUD and suicides without this comorbidity, genes involved in this process were detected among the probes influenced by the interaction between SUD and completed suicide, including CNOT, ATP6VOC, $M A R C K S L 1, N D R G 1$, and RAN, suggesting that this process may be relevant in the interaction of both disorders. The overexpression of genes that participate in the differentiation of glial cells, such as SOX10, OLIG1, NDRG1, CNP, and PTPN11, may be potentially relevant in suicide with SUD. Suicide subjects with alcohol dependence have been shown to present higher glial density in the anterior cingulate cortex compared with suicide subjects without dependence [56].

Although we did not evaluate gene expression in this specific brain area, these results indicate that substances, such as alcohol, in interaction with completed suicide may influence glial density. A possible mechanism that could contribute to the increase in the expression of genes related to glial proliferation is the combination of immunomodulatory effects and factors related to stress, which would lead to higher glial density [56]. This also coincides with the GO categories of other genes that participate in the interaction of both disorders, such as the response to stress and the regulation of immune system processes.

It has been suggested that the interaction between depression and SUD could lead to glial dysfunction and a consequent alteration in glutamatergic neurotransmission $[57,58]$, so the pharmacological modulation of this circuit may play a key role in the treatment of SUD in comorbidity with other mental disorders. Ketamine, a glutamate-modulating agent, has been shown to reduce suicide in high-risk populations [58-60], so the inclusion of this medication in the treatment of subjects with SUD with suicidal tendencies is an option that would be worth exploring.

It should be noted that this is the first study to carry out a transcriptome analysis of suicide in a Latino population. Suicide is a phenomenon where different individual, sociodemographic, and situational factors interact, and these factors can vary according to place and ethnicity. The environmental factors to which subjects in the Mexican population are exposed may lead to epigenetic changes that are different from those of other popula- 
tions, and this could lead to unique influences on gene expression.

The present study has some limitations, such as the relatively small sample size, especially in the nonsuicidal groups; it would be desirable to increase the sample number and include more subjects with abuse to substances other than alcohol, so we could explore the effect of these substances in interaction with suicide. Another limitation is the lack of analysis in other brain regions potentially relevant for suicide and SUD. Future studies should focus on epigenetic changes and/or genotype changes that might be underlying the transcriptome alterations observed in this study, as well as the inclusion of other potential confounding clinical characteristics not examined in this study (for instance, medications and co-occurring drug dependence not detected by our methods).

\section{Conclusion}

Our study suggests that suicide with SUD presents alterations in gene expression that are different from those of suicide without this comorbidity and nonsuicide subjects with SUD. Therefore, our results suggest that the interaction of suicide with SUD leads to a unique expression profile. Our results also suggest an alteration in the expression of genes implicated in glial differentiation and glutamatergic neurotransmission in suicides with SUD.

\section{Acknowledgments}

We thank Dr. Simon Wallace for his appreciable help in the language editing and proofreading of this paper.

\section{Statement of Ethics}

The Ethics Committee for Human Research at the National Institute of Genomic Medicine approved this research (CEI 2016/33).

\section{Disclosure Statement}

The authors have no conflicts of interest to declare.

\section{Funding Sources}

This work was funded by a grant from the National Council for Science and Technology (CONACyT: \#233695)

\section{Author Contributions}

B.C. participated in the conception of the work, sample processing, analysis and interpretation of the data. She also wrote the present paper; this work is part of her $\mathrm{PhD}$ program. N.M.-J. and G.R.F. participated in the design of the work, sample processing, acquisition of the data (microarrays), and results interpretation. In addition, they revised the present manuscript, providing essential ideas, and approved its final version. R.C.M.-M., F.G.-D., A.M.-L., and C.D.-O. participated in the sample acquisition and revised the present paper, providing relevant content. C.F. contributed to the data analysis, results interpretation, and writing of the present paper. C.W.-B., D.C.G., H.N., and P.O.-W. contributed to the conception and design of the work, and interpretation of the data. They participated actively in the writing of the present paper and provided key ideas for results interpretation. All the authors revised and approved the final version of the manuscript. All authors agree to be accountable for all aspects of the work in ensuring that questions related to the accuracy and integrity of any part of the work are appropriately investigated and resolved.

\section{References}

1 World Health Organization. Preventing suicide: a global imperative. 2015 [accessed 2018 Mar 18]. Available from: http://www.who.int/ mental_health/suicide-prevention/world_report_2014/en/.

2 Instituto Nacional de Estadística y Geografía. Estadísticas a propósito del día mundial para la prevención del suicidio. 2017. Available from: http://www.inegi.org.mx/saladeprensa/aproposito/2017/suicidios2017_Nal.pdf.

3 Haw C, Hawton K, Niedzwiedz C, Platt S. Suicide clusters: a review of risk factors and mechanisms. Suicide Life Threat Behav. 2013 Feb;43(1):97-108.
4 Fleischmann A, Bertolote JM, Belfer M, Beautrais A. Completed suicide and psychiatric diagnoses in young people: a critical examination of the evidence. Am J Orthopsychiatry. 2005 Oct;75(4):676-83.

5 Cho SE, Na KS, Cho SJ, Im JS, Kang SG. Geographical and temporal variations in the prevalence of mental disorders in suicide: systematic review and meta-analysis. J Affect Disord. 2016 Jan; 190:704-13.

6 Turecki G, Brent DA. Suicide and suicidal behaviour. Lancet. 2016 Mar;387(10024):122739.
7 Wilcox HC, Conner KR, Caine ED. Association of alcohol and drug use disorders and completed suicide: an empirical review of cohort studies. Drug Alcohol Depend. 2004 Dec;76 Suppl:S11-9.

8 Borges G, Walters EE, Kessler RC. Associations of substance use, abuse, and dependence with subsequent suicidal behavior. Am J Epidemiol. 2000 Apr;151(8):781-9.

9 Miller M, Borges G, Orozco R, Mukamal K, Rimm EB, Benjet C, et al. Exposure to alcohol, drugs and tobacco and the risk of subsequent suicidality: findings from the Mexican Adolescent Mental Health Survey. Drug Alcohol Depend. 2011 Jan;113(2-3):110-7. 
10 Fiori LM, Turecki G. Gene expression profiling of suicide completers. Eur Psychiatry. 2010 Jun;25(5):287-90.

11 Gandal MJ, Haney JR, Parikshak NN, Leppa V, Ramaswami G, Hartl C, et al.; CommonMind Consortium; PsychENCODE Consortium; iPSYCH-BROAD Working Group Shared molecular neuropathology across major psychiatric disorders parallels polygenic overlap. Science. 2018 Feb;359(6376):693-7.

12 Raust A, Slama F, Mathieu F, Roy I, Chenu A, Koncke D, et al. Prefrontal cortex dysfunction in patients with suicidal behavior. Psychol Med. 2007 Mar;37(3):411-9.

13 Richard-Devantoy S, Berlim MT, Jollant F. A meta-analysis of neuropsychological markers of vulnerability to suicidal behavior in mood disorders. Psychol Med. 2014 Jun;44(8): 1663-73.

14 Goldstein RZ, Volkow ND. Dysfunction of the prefrontal cortex in addiction: neuroimaging findings and clinical implications. Nat Rev Neurosci. 2011 Oct;12(11):652-69.

15 Romero-Pimentel AL, et al. Socio-demographic and clinical characteristics of completed suicides in Mexico City 2014-2015. Front Psychiatry. 2018 Sep 7;9:402.

16 Dunning M, Lynch A, Eldridge M. illuminaHumanv4.db: Illumina HumanHT12v4 annotation data (chip illuminaHumanv4). 2015. $\mathrm{R}$ package version 1.26.0.

17 Ritchie ME, Phipson B, Wu D, Hu Y, Law CW, Shi W, et al. limma powers differential expression analyses for RNA-sequencing and microarray studies. Nucleic Acids Res. 2015 Apr;43(7):e47.

18 Phipson B, Lee S, Majewski IJ, Alexander WS, Smyth GK. Robust hyperparameter estimation protects against hypervariable genes and improves power to detect differential expression. Ann Appl Stat. 2016 Jun;10(2):946-63.

19 Silver JD, Ritchie ME, Smyth GK. Microarray background correction: maximum likelihood estimation for the normal-exponential convolution. Biostatistics. 2009 Apr;10(2):352-63.

20 Smyth GK, Speed T. Normalization of cDNA microarray data. Methods. 2003 Dec;31(4): 265-73.

21 Nueda MJ, Ferrer A, Conesa A. ARSyN: a method for the identification and removal of systematic noise in multifactorial time course microarray experiments. Biostatistics. 2012 Jul;13(3):553-66.

22 Benjamini Y, Hochberg Y. Controlling the false discovery rate: a practical and powerful approach to multiple testing. J R Stat Soc Series B Stat Methodol. 1995;57:289-300.

23 Warnes GR, Bolker B, Bonebakker L, Gentleman R, Huber W, Liaw A, Lumley T, Maechler M, Magnusson A, Moeller S, Schwartz M, Venables B: R package gplots. 2014. Available from: http://cran.r-project.org/web/packages/gplots/gplots.pdf.

24 R Core Team. R: A Language and Environment for StatisticalComputing. Vienna: $\mathrm{R}$ Foundation for Statistical Computing; 2013. Available from: https://www.r-project.org/.
25 Huang W, Sherman BT, Lempicki RA. Systematic and integrative analysis of large gene lists using DAVID bioinformatics resources. Nat Protoc. 2009;4(1):44-57.

26 Huang W, Sherman BT, Lempicki RA. Bioinformatics enrichment tools: paths toward the comprehensive functional analysis of large gene lists. Nucleic Acids Res. 2009 Jan;37(1): $1-13$.

27 Hong G, Zhang W, Li H, Shen X, Guo Z. Separate enrichment analysis of pathways for upand downregulated genes. J R Soc Interface. 2013 Dec;11(92):20130950.

28 Hosack DA, Dennis G Jr, Sherman BT, Lane HC, Lempicki RA. Identifying biological themes within lists of genes with EASE. Genome Biol. 2003;4(10):R70.

29 Krämer A, Green J, Pollard J Jr, Tugendreich S. Causal analysis approaches in Ingenuity Pathway Analysis. Bioinformatics. 2014 Feb; 30(4):523-30.

30 Klempan TA, Sequeira A, Canetti L, Lalovic A, Ernst C, ffrench-Mullen J, et al. Altered expression of genes involved in ATP biosynthesis and GABAergic neurotransmission in the ventral prefrontal cortex of suicides with and without major depression. Mol Psychiatry. 2009 Feb;14(2):175-89.

31 Sequeira A, Klempan T, Canetti L, ffrenchMullen J, Benkelfat C, Rouleau GA, et al. Patterns of gene expression in the limbic system of suicides with and without major depression. Mol Psychiatry. 2007 Jul;12(7):640-55.

32 Kim S, Choi KH, Baykiz AF, Gershenfeld HK. Suicide candidate genes associated with bipolar disorder and schizophrenia: an exploratory gene expression profiling analysis of postmortem prefrontal cortex. BMC Genomics. 2007 Nov;8(1):413

33 Ernst C, Nagy C, Kim S, Yang JP, Deng X, Hellstrom IC, et al. Dysfunction of astrocyte connexins 30 and 43 in dorsal lateral prefrontal cortex of suicide completers. Biol Psychiatry. 2011 Aug;70(4):312-9.

34 Karnoub AE, Der CJ. Rho Family GTPases and Cellular Transformation. Madame Curie Bioscience Database Austin (TX): Landes Bioscience; p. 2000-13. Available from: https://www.ncbi.nlm.nih.gov/books/ NBK6594/.

35 Karunakaran I, van Echten-Deckert G. Sphingosine 1-phosphate - A double edged sword in the brain. Biochim Biophys Acta Biomembr. 2017 Sep;1859(9 9 Pt B):1573-82.

36 Li YX, Yu ZW, Jiang T, Shao LW, Liu Y, Li N, et al. SNCA, a novel biomarker for Group 4 medulloblastomas, can inhibit tumor invasion and induce apoptosis. Cancer Sci. 2018 Apr;109(4):1263-75.

37 Johansson S, Ekström TJ, Marinova Z, Okvist A, Sheedy D, Garrick T, et al. Dysregulation of cell death machinery in the prefrontal cortex of human alcoholics. Int J Neuropsychopharmacol. $2009 \mathrm{Feb}$;12(1):109-15.

38 Harper C, Dixon G, Sheedy D, Garrick T. Neuropathological alterations in alcoholic brains. Studies arising from the New South
Wales Tissue Resource Centre. Prog Neuropsychopharmacol Biol Psychiatry. 2003 Sep; 27(6):951-61.

39 Miguel-Hidalgo JJ, Wei J, Andrew M, Overholser JC, Jurjus G, Stockmeier CA, et al. Glia pathology in the prefrontal cortex in alcohol dependence with and without depressive symptoms. Biol Psychiatry. 2002 Dec;52(12): 1121-33.

40 Underwood MD, Kassir SA, Bakalian MJ, Galfalvy H, Mann JJ, Arango V. Neuron Density and Serotonin Receptor Binding in Prefrontal Cortex in Suicide. Int J Neuropsychopharmacol. 2012 May;15(4):435-47.

41 Schnieder TP, Trencevska I, Rosoklija G Stankov A, Mann JJ, Smiley J, et al. Microglia of prefrontal white matter in suicide. J Neuropathol Exp Neurol. 2014 Sep;73(9):880-90.

42 Sequeira A, Mamdani F, Ernst C, Vawter MP Bunney WE, Lebel V, et al. Global brain gene expression analysis links glutamatergic and GABAergic alterations to suicide and major depression. PLoS One. 2009 Aug;4(8):e6585.

43 Choudary PV, Molnar M, Evans SJ, Tomita $\mathrm{H}$, Li JZ, Vawter MP, et al. Altered cortical glutamatergic and GABAergic signal transmission with glial involvement in depression. Proc Natl Acad Sci USA. 2005 Oct;102(43): 15653-8.

44 Chandler LJ, Newsom H, Sumners C, Crews F. Chronic ethanol exposure potentiates NMDA excitotoxicity in cerebral cortical neurons. J Neurochem. 1993 Apr;60(4):1578-81.

45 Selim M, Bradberry CW. Effect of ethanol on extracellular 5-HT and glutamate in the nucleus accumbens and prefrontal cortex: comparison between the Lewis and Fischer 344 rat strains. Brain Res. 1996 Apr;716(1-2):157-64.

46 Miguel-Hidalgo JJ, Wilson BA, Hussain S, Meshram A, Rajkowska G, Stockmeier CA. Reduced connexin 43 immunolabeling in the orbitofrontal cortex in alcohol dependence and depression. J Psychiatr Res. 2014 Aug;55: 101-9.

47 Beech RD, Lowthert L, Leffert JJ, Mason PN, Taylor MM, Umlauf S, et al. Increased peripheral blood expression of electron transport chain genes in bipolar depression. Bipolar Disord. 2010 Dec;12(8):813-24.

48 Austin CD, Lawrence DA, Peden AA, Varfolomeev EE, Totpal K, De Mazière AM, et al. Death-receptor activation halts clathrin-dependent endocytosis. Proc Natl Acad Sci USA. 2006 Jul;103(27):10283-8

49 Lin-Hendel. Erika G. Differential Requirements For Mitochondria During Neuronal Migration. 2017. Publicly Accessible Penn Dissertations. 2424. Available from: https:// repository.upenn.edu/edissertations/2424.

50 Devaraju P, Zakharenko SS. Mitochondria in complex psychiatric disorders: Lessons from mouse models of 22q11.2 deletion syndrome: Hemizygous deletion of several mitochondrial genes in the 22q11.2 genomic region can lead to symptoms associated with neuropsychiatric disease. BioEssays. 2017 Feb;39(2): 1600177. 
51 Shieh JC, Schaar BT, Srinivasan K, Brodsky FM, McConnell SK. Endocytosis regulates cell soma translocation and the distribution of adhesion proteins in migrating neurons. PLoS One. 2011 Mar;6(3):e17802.

52 Sun Y, Deng Y, Xiao M, Hu L, Li Z, Chen C. Chondroitin sulfate proteoglycans inhibit the migration and differentiation of oligodendrocyte precursor cells and its counteractive interaction with laminin. Int J Mol Med. 2017 Dec;40(6):1657-68.

53 Uribe E, Wix R. Neuronal migration, apoptosis and bipolar disorder. Rev Psiquiatr Salud Ment. 2012 Apr-Jun;5(2):127-33. English, Spanish.
54 Pantazatos SP, Huang YY, Rosoklija GB, Dwork AJ, Arango V, Mann JJ. Whole-transcriptome brain expression and exon-usage profiling in major depression and suicide: evidence for altered glial, endothelial and ATPase activity. Mol Psychiatry. 2017 May; 22(5):760-73.

55 Costa V, Esposito R, Aprile M, Ciccodicola A. Non-coding RNA and pseudogenes in neurodegenerative diseases: "The (un)Usual Suspects". Front Genet. 2012 Oct;3:231.

56 Hercher C, Parent M, Flores C, Canetti L, Turecki G, Mechawar N. Alcohol dependencerelated increase of glial cell density in the anterior cingulate cortex of suicide completers. J Psychiatry Neurosci. 2009 Jul;34(4):281-8.

57 Niciu MJ, Henter ID, Sanacora G, Zarate CA Jr. Glial abnormalities in substance use disorders and depression: does shared glutamatergic dysfunction contribute to comorbidity? World J Biol Psychiatry. 2014 Jan;15(1):2-16.
58 Goodwani S, Saternos H, Alasmari F, Sari Y. Metabotropic and ionotropic glutamate receptors as potential targets for the treatment of alcohol use disorder. Neurosci Biobehav Rev. 2017 Jun;77:14-31.

59 Price RB, Nock MK, Charney DS, Mathew SJ. Effects of intravenous ketamine on explicit and implicit measures of suicidality in treatment-resistant depression. Biol Psychiatry. 2009 Sep;66(5):522-6.

60 DiazGranados N, Ibrahim LA, Brutsche NE, Ameli R, Henter ID, Luckenbaugh DA, et al. Rapid resolution of suicidal ideation after a single infusion of an N-methyl-D-aspartate antagonist in patients with treatment-resistant major depressive disorder. J Clin Psychiatry. 2010 Dec;71(12):1605-11. 